\title{
The first reported case of tuberculosis of the glans penis in Sri Lanka
}

\author{
Perera $\mathrm{HP}^{1}$, Ilangarathne $\mathrm{A}^{2}$, Masiama $\mathrm{MNN}^{3}$, Perera $\mathrm{O}^{4}$, Murugadas $\mathrm{N}^{5}$
}

\begin{abstract}
Sri Lanka has a low prevalence for tuberculosis (TB). Though pulmonary $\mathrm{TB}$ is the commonest presentation, in immune compromised individuals extra-pulmonary sites are involved. Here we report a case of extra- pulmonary TB occurring in genitalia in a patient who is immune competent.
\end{abstract}

Key words: Extra pulmonary tuberculosis, Mycobacterium tuberculosis, Acid fast bacilli

\section{Introduction}

Tuberculosis is a chronic inflammatory disease caused by Mycobacterium tuberculosis, which is acquired through droplet inhalation. Early diagnosis and treatment is vital in preventing dissemination. Lungs are the most commonly affected organ by TB. However, extra pulmonary TB involving other organs occurs specially among immunocompromised persons. Genital disease is a rare form of extra pulmonary tuberculosis, affecting middle aged men with renal or pulmonary tuberculosis. ${ }^{1}$ The first case of TB in the glans penis was reported by Hellerstrom and Bafverstedt in 1988. ${ }^{2} \mathrm{~TB}$ of glans penis presents as a superficial lesion which is difficult to differentiate from malignancy. ${ }^{3}$ The glans penis can be involved primarily as an ulcerative lesion, by extension via the urethra or via haematogenous spread. ${ }^{4}$ The standard six months course of anti TB drugs is sufficient to treat the disease. We report a case of secondary TB in the glans penis.

\section{Case Report}

A 32 years un married male labourer was referred to Horana sexual transmitted disease clinic from outpatient department with a history of a non-healing painless growth on the penis of six months duration. $\mathrm{He}$ had no past history of genital ulcers. He complained of evening pyrexia and cough for two weeks. He had loss of weight for the past two months. He denied any form of sexual exposures or illicit drug use. He was an ex-smoker. He lives with parents in a house with adequate ventilation and light.

Examination revealed a non-tender granulomatous lesion on the glans penis covering the urethral meatus (figure1).He had no ulcers elsewhere. There was no lymphadenopathy. He did not have a BCG scar. There was no hepatosplenomegaly. Examination of lungs revealed bronchial breathing in both upper lobes . Rest of the systems was normal.

Chest X-ray showed milliary mottling in both upper lobes (figure 2). Venereal Disease Research Laboratory test (VDRL), Treponema Pallidum Particle Agglutination test (TPPA), Human Immune Deficiency Virus antibody test and Hepatitis B Surface antigens were negative. Urine full report, liver function tests, and renal function tests were also normal. He had an ESR of $85 \mathrm{~mm}$. There was a Mantoux reaction of $13 \mathrm{~mm}$. Full blood count showed a WBC of $8.8 \times 10^{3}$, with N-73\% L-23\% E-03\% M$02 \%$. RBC-4.0x $10^{5} / \mathrm{L}, \mathrm{Hb}-12.0 \mathrm{~g} \%$ and platelets $390 \times 10^{3} /$ uL. Direct smear from the lesion was negative for $\mathrm{AFB}$, but $\mathrm{TB}$ culture taken from the penile ulcer was positive for acid fast bacilli (AFB).Dark ground microscopy of smear from the lesion was negative for Treponemapallidum. Tzanck smear was negative for giant cells. Biopsy taken from the lesion was suggestive of tuberculosis.

${ }^{1}$ Consultant Venereologist, ${ }^{2}$ Medical officer ,STDClinic Kalutara, ${ }^{3}$ Consultant Chest Physician District General Hospital Kalutara, ${ }^{4}$ Consultant Pathologist, ${ }^{5}$ Consultant Surgeon Base Hospital Horana 

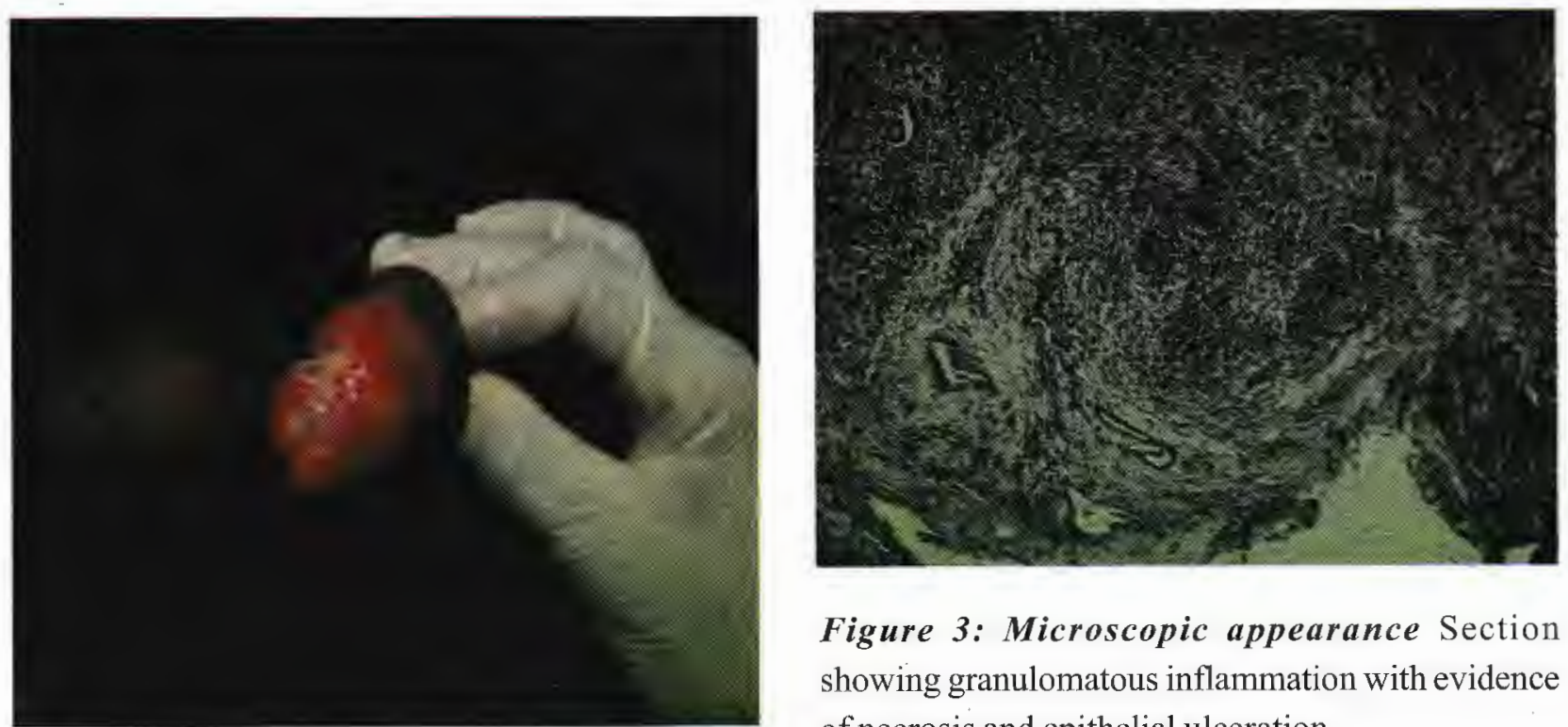

Figure 3: Microscopic appearance Section showing granulomatous inflammation with evidence of necrosis and epithelial ulceration

Figure1A granulomatous lesion on his penis
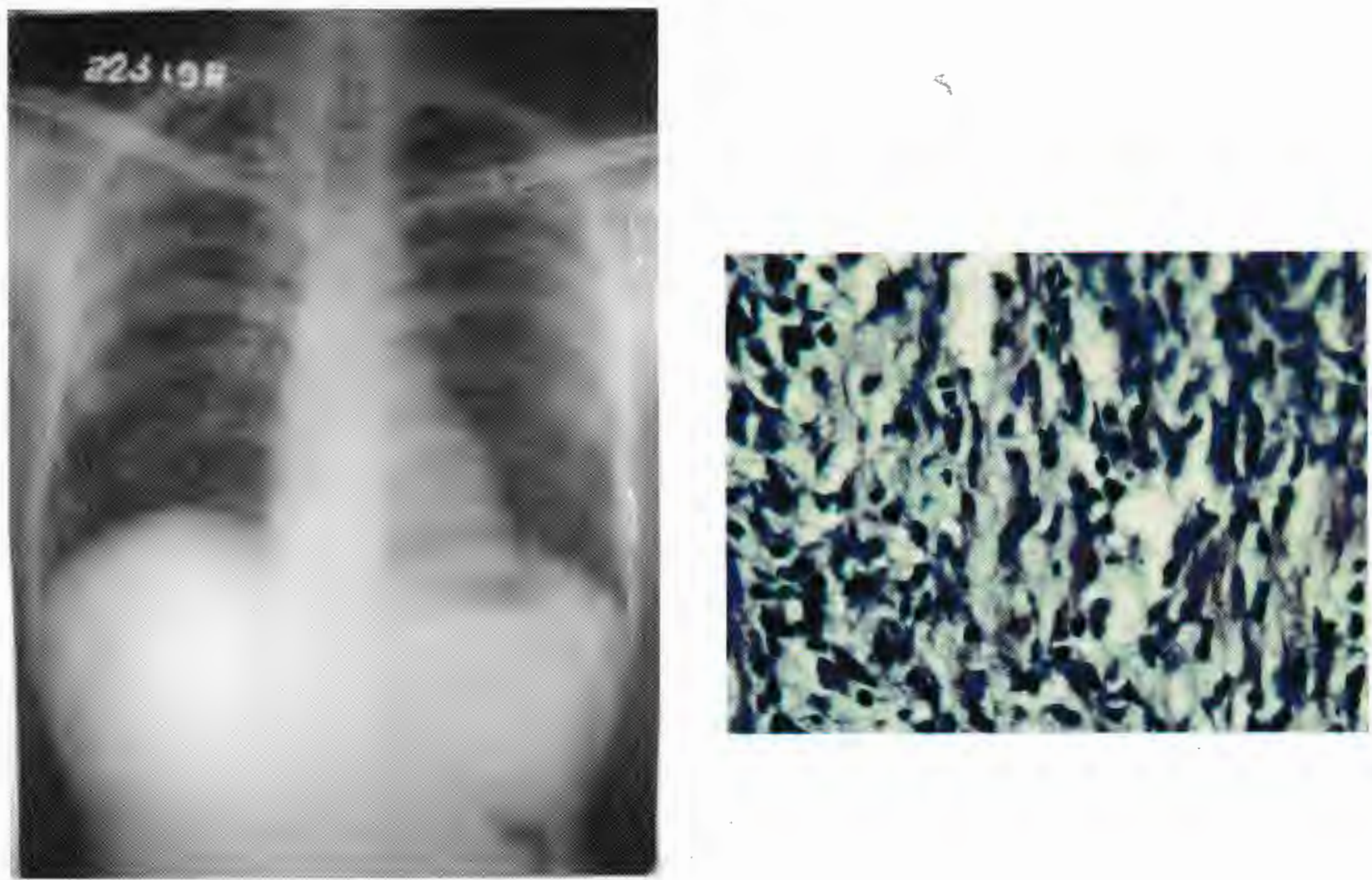

Figure 2 Chest radiograph of the patient

Figure 4 Microscopic appearance Section showing plasma cells, neutrophils, lymphocytes, epitheloid histiocytes and Langerhan type giant cells (H\&E $\mathrm{x} 200$ ) 
Microscopy showed tissue infiltrated by numerous histiocytes, plasma cells, neutrophils and lymphocytes. Several granulomatous areas were seen containing epitheloid histiocytes and Langerhan typegiant cells. Epithelial ulceration and focal necrosis were also present. The blood vessels showed marked endothelial cell swelling and proliferation and infiltration of wall by inflammation cells (end arteritis obliterans' like lesion).He had three consecutive sputum samples positive for AFB indicating having TB in both lungs and glans TB.

\section{Discussion}

He was started on anti tuberculous therapy with isoniazid, rifampicin, ethambutol and pyrazinamide for four months, and isoniazid and rifampicin for 2 months. After two weeks of treatment, the lesions on the glans reduced significantly and were healed completely by six weeks. The response to treatment was monitored with sputum for AFB at three months and five months after which were negative.

A similar histological appearance could be seen in late secondary syphilis, but negative VDRL and TPHA results excluded this. Though rare, a papulonecrotic lesion on glans should raise the suspicion of TB. Investigations for TB should be carried out even when there is no clear history of TB. Biopsy from such a lesion is also mandatory as malignancy is also a possibility.

\section{References}

1.Jacob JT, Nguyen TM, RaySM.Male genital tuberculosis.Lancet InfectiousDisease. 2008 May; 8(5):335-42

\section{Jeyakumar W, Ganesh R, Mohanram MS} ,Shanmugasundararaj A.Papulonecrotic tuberculosis of the glans penis. Genitourinary Medicine.1988;64:130-2

3. Nishigori C, Taniguchi S, Hayakawa M, Imamura S.Penis tuberculosis:papulonecrotic tuberculosis on the glanspenis.
4. Johnson WDJr, Johnson CW, LoweFC.Tuberculosis and parasitic disease of the genitourinary system.Campbel'sUrology. $8^{\text {th }}$ ed Philadelphia 2002.p750-1 